\title{
Differential infection outcome of Chlamydia trachomatis in human blood monocytes and monocyte-derived dendritic cells
}

\author{
Baishakhi Datta*, Florence Njau, Jessica Thalmann, Hermann Haller and Annette D Wagner
}

\begin{abstract}
Background: Chlamydia trachomatis is an intracellular bacteria which consist of three biovariants; trachoma (serovars A-C), urogenital (serovars D-K) and lymphogranuloma venereum (L1-L3), causing a wide spectrum of disease in humans. Monocytes are considered to disseminate this pathogen throughout the body while dendritic cells (DCs) play an important role in mediating immune response against bacterial infection. To determine the fate of $C$. trachomatis within human peripheral blood monocytes and monocyte-derived DCs, these two sets of immune cells were infected with serovars $\mathrm{Ba}, \mathrm{D}$ and $\mathrm{L} 2$, representative of the three biovariants of $\mathrm{C}$. trachomatis.

Results: Our study revealed that the different serovars primarily infect monocytes and DCs in a comparable fashion, however undergo differential infection outcome, serovar L2 being the only candidate to inflict active infection. Moreover, the $C$. trachomatis serovars Ba and D become persistent in monocytes while the serovars predominantly suffer degradation within DCs. Effects of persistence gene Indoleamine 2, 3-dioxygenase (IDO) was not clearly evident in the differential infection outcome. The heightened levels of inflammatory cytokines secreted by the chlamydial infection in DCs compared to monocytes seemed to be instrumental for this consequence. The immune genes induced in monocytes and DCs against chlamydial infection involves a different set of Toll-like receptors, indicating that distinct intracellular signalling pathways are adopted for immune response.
\end{abstract}

Conclusion: Our results demonstrate that the host pathogen interaction in chlamydia infection is not only serovar specific but manifests cell specific features, inducing separate immune response cascade in monocytes and DCs.

Keywords: Chlamydia trachomatis, Monocyte, Dendritic cell, Cytokine, Gene expression

\section{Background}

Chlamydia trachomatis is an obligate intracellular pathogen with unique biphasic developmental cycle alternating between the infectious elementary body (EB) and the metabolically active reticulate body (RB). Based on the antigenic variation of the major outer membrane protein (MOMP), the C. trachomatis isolates have been divided into three different biovariants [1]. Serovars A, B, Ba and $C$ cause ocular infections, currently infecting 150 million people worldwide [2,3]; serovars D, E, F, G, H, I, J and K cause sexually transmitted disease with more than 90 million new cases of genital infections occurring every year $[4,5]$ and serovars L1, L2 and L3 cause lymphgranuloma

\footnotetext{
*Correspondence: baishakhid2004@gmail.com

Department of Nephrology, Hannover Medical School, Hannover, Germany
}

venereum (LGV) primarily affecting the lymphatic system with recent outbreaks in Western Europe [6,7]. Comparative genomic studies demonstrate that the genome of $C$. trachomatis serovars are strikingly similar to each other and share more than $99 \%$ identity $[8,9]$. Genetic differences were observed centring around the plasticity zone i.e. $\sim 50 \mathrm{~kb}$ region near the predicted termination origin of the genome, experiencing a higher degree of DNA arrangement [10], MOMP and members of polymorphic membrane protein (pmp) gene family [11]. However the occurrence of quantitatively different infections by different serovars within a given host has been intriguing. In vivo studies infecting mice intranasally [12] or intravaginally [13] with different serovars of $C$. trachomatis has revealed a great deal of variation in infection kinetics. Genome analysis could reveal that a functional tryptophan 
synthase enzyme and toxin might be the principal virulence factors underlying this distinct tropism in terms of organ specific disease termed as organotropism [14]. Studies including LGV serovars confirmed the observation that the variability resided mainly in the plasticity zone [15].

Chlamydia primarily targets the host epithelial cells and resides within distinct membrane bound vacuoles termed as chlamydial inclusion. The chlamydia proliferate within inclusion and inhibits their acidification by avoiding fusion with lysosomal compartments $[16,17]$. However the association of $C$. trachomatis with reactive arthritis have raised questions how chlamydia is transported from the site of infection to the site of inflammatory disease in the joints or vasculature [18-20]. Studies have shown that the $C$. trachomatis infection is characterized by infiltration with polymorphonuclear leukocyte (PMNs) in the acute phase and mononuclear cells in the chronic phase [21]. Hence there have been suggestions that circulating monocytes delivers the pathogen to other organs and initiate immunological response and inflammation. The role of $C$. pneumoniae infected monocytes as a vector, transmitting the pathogen to vascular wall, has been elucidated earlier [22]. The C. trachomatis infection of monocytes in vitro, have mostly resulted in noncultivable state in which the bacteria although metabolically active could not produce active infectious particle when recultured in HeLa cells [23,24].

Dendritic cells (DCs) are the first professional antigen presenting cells encountering the bacteria after initial infection. DCs are very efficient in processing and presenting bacterial antigens and play a crucial role in activating $\mathrm{T}$ cell-dependent immune response [25,26]. Studies have illustrated the role of DCs to evoke strong immune responses against chlamydial infections by stimulating $\mathrm{T}$ cell reaction $[27,28]$. There are contrasting evidences of the fate of $C$. trachomatis within DCs; there has been observations that C. trachomatis inclusion fuses with lysosomal compartment [29] while another study confirmed that the chlamydial inclusion did not colocalize with lysosome associated membrane protein (Lamp) 1 or Major histocompatibility complex (MHC) II compartments [30]. C. trachomatis infection of DCs was characterized by upregulation of co-stimulatory molecules and secretion of inflammatory cytokines [31]. Previous studies have implicated cytokines IFN- $\gamma$ as well TNF of inducing indoleamine 2,3-dioxygenase (IDO), an enzyme catalysing the degradation of tryptophan leading to chlamydial growth arrest [32-34]. The presence of a functional tryptophan synthase in the urogenital serovars while its absence in the ocular serovars $[35,36]$ has been considered to be pivotal. The genital serovars survive by utilizing indole produced by vaginal microbial flora as a substrate for tryptophan synthesis in IDO induced tryptophan-depleted culture medium [37].
However, little is known about the growth characteristics of the different biovariants of $C$. trachomatis in monocytes and DCs -the two major immune cells that the bacterium encounters during infection. Hence we selected three serovars $\mathrm{Ba}, \mathrm{D}$ and L2; representative of the ocular, urogenital and lymphogranuloma serovars respectively, for comparative study in human monocytes and monocyte- derived DCs. In our study we observed the chlamydial morphology within infected monocytes and DCs; analyzed their metabolic activity and could illustrate the cytokine induced inflammatory response. We were also able to propose the distinct immune response pathways employed by $C$. trachomatis infected monocytes and DCs.

\section{Methods}

\section{Chlamydia culture}

Chlamydia trachomatis serovars D/UW-3/Cx(ATCCVR885) and serotype LGV II strain 434(ATCC-VR902B) were kindly provided by Prof Andreas Klos (Medical Microbiology and Hospital Epidemiology, Hannover Medical School, Germany) and Chlamydia trachomatis serotype Ba Apache-2(ATCC-VR347) was kindly sent by Prof Eberhard Straube (Institute of Medical Microbiology, Friedrich Schiller University of Jena, Jena, Germany).

Bacterial stocks were prepared as described previously [38]. Briefly, HeLa cell monolayers infected with C. trachomatis serovar Ba, D and L2 EBs were cultivated at $37^{\circ} \mathrm{C}$ and $5 \% \mathrm{CO}_{2}$ in Earle's MEM containing glutamine, supplemented with $10 \%$ fetal calf serum (FCS), $0.1 \mathrm{M}$ nonessential amino acids, and $1 \mathrm{mM}$ sodium pyruvate (PAA Laboratories, Pasching, Germany) along with $1 \mu \mathrm{g} / \mathrm{ml}$ cycloheximide (Sigma-Aldrich, Steinheim, Germany). EBs from infected cells were harvested at 48 hours (Serovar L2) to 72 hours (Serovar Ba and Serovar D) p.i., purified by 2 step ultracentrifugation and collected in transport medium (1x PBS, including 6.86\% saccharose, $40 \mu \mathrm{g} / \mathrm{ml}$ Gentamicin, $0.002 \%$ Phenol red, 2\% FCS). The final stock was stored in small aliquots in transport medium at $-80^{\circ} \mathrm{C}$ until use. Mock control was prepared following the complete propagation, harvest and purification procedure for EBs in the absence of $C$. trachomatis infection. All the stocks were free of Mycoplasma as tested by Venor GeM kit (Minerva Biolabs, Berlin, Germany). To quantify the $\mathrm{EB}$, the inclusions were counted and the EB determined as inclusion-forming-units (IFU)/ml. For heat inactivation, EBs of C. trachomatis serovars Ba, D and L2 were treated at $75^{\circ} \mathrm{C}$ for 30 minutes. All the plastic wares were obtained from Greiner Bio-One (Greiner Bio-One GmbH, Frickenhausen, Germany) unless otherwise mentioned.

\section{Culture of monocytes and monocyte-derived DCs}

Heparinized buffy coats from healthy blood donors were obtained from Blutspendedienst NSTOB Springe, Bremen, 
Germany. Buffy coats were prepared from whole blood collected from volunteer donors under informed consent according to the current German hemotherapy guidelines [39]. Peripheral blood mononuclear cells (PBMCs) were isolated by Ficoll-Hypaque density gradient centrifugation using Lymphocyte Separation Medium (PAA Laboratories, Pasching, Germany). For each experiment a different blood donor was used. Monocytes were isolated by negative selection using the Monocyte Isolation kit II (Miltenyi Biotec GmbH, Bergisch Gladbach, Germany) according to manufacturer's protocol (monocyte purity $>90 \%$ ).

Monocytes were seeded on Poly L-Lysine $(0.01 \%)$ coated 24-well plate at a density of $3 \times 10^{5}$, allowed to adhere for 2 hours before infection and cultured in RPMI-1640 (PAA Laboratories, Pasching, Germany) containing 10\% FCS. For DCs, $3 \times 10^{5}$ monocytes were cultured in RPMI1640 medium with autologous serum in 24-well plate for 7 days in the presence of IL-4 $(1000 \mathrm{U} / \mathrm{mL})$ (R\&D Systems, Wiesbaden, Germany) and GM-CSF (500 U/mL) (Novartis Pharma, Nurnberg, Germany) as described previously [40].

\section{Infection of monocyte and monocyte-derived DC}

Monocytes and the monocyte-derived DCs were infected with C. trachomatis serovars $\mathrm{Ba}, \mathrm{D}$ and $\mathrm{L} 2$ at a multiplicity of infection (MOI) of 3 by centrifugation for $30 \mathrm{~min}$ at $400 \times g$ with further incubation for $30 \mathrm{~min}$ at $37^{\circ} \mathrm{C}$ in $5 \% \mathrm{CO}_{2}$. Following incubation, the cells were washed with phosphate-buffered saline (PBS) to remove extracellular bacteria. Fresh RPMI-1640 containing 10\% FCS was added to infected monocytes and RPMI-1640 with autologous serum was added to infected DCs. The infected cultures were incubated at $37^{\circ} \mathrm{C}$ and $5 \% \mathrm{CO}_{2}$ for intended durations. For immunofluorescence, cells were grown on coverslips. Infected cells were harvested by rubber scraper at different time points as per experimental protocol. The cell pellets for PCR/reinfection as well as supernatants for cytokine analysis were stored at $-80^{\circ} \mathrm{C}$. Mock infected controls were prepared for every set of experiment to assess the contribution of host cell debris. Control samples were routinely checked for the presence of chlamydia antigens in the donor samples by immunofluorescence microscopy.

\section{Immunofluorescence microscopy}

The infected monocytes and DCs after intended incubation were fixed in $2 \%$ para-formaldehyde for $10 \mathrm{~min}$ and washed 3 times in PBS. Cells were permeabilized with $0.5 \%$ TritonX-100 for 3 minutes. Following fixation, the cells were blocked with PBS containing 1\% BSA and 1\% FCS. Genus-specific fluorescein isothiocyanate (FITC)labelled monoclonal antibody (Pathfinder Chlamydia Confirmation System; Bio-Rad, Redmond, WA) was used to stain the chlamydial inclusions, while the monocytes and
DCs were counterstained with Evan's Blue at room temperature for $45 \mathrm{~min}$. The samples were then washed once with PBS and then washed twice with PBS/DAPI (1:2500) to stain the cell nuclei. Images were captured in 10 random fields with a fluorescence microscope (Leica DMLB, Germany) with standard filters at $63 \mathrm{X}$ magnification. ImageJ was used to count the number of inclusions/ cells in replicate samples. Data from 3 independent experiments were combined to calculate the mean and standard deviation.

\section{Analysis of the infectivity of $C$. trachomatis in monocytes/DCs}

Cells harvested at different time points were lysed in an ultrasonic sonicator bath (Jürgen's Hannover, Germany). Cell lysates were used to infect HeLa cells seeded on coverslips and cultured in MEM media containing $1 \mu \mathrm{g} / \mathrm{ml}$ cycloheximide at $37^{\circ} \mathrm{C}$ in $5 \% \mathrm{CO}_{2}$ for the intended duration. At the end of the infection period, cells were fixed for $10 \mathrm{~min}$ in absolute methanol, air-dried, and stained using FITC-labelled monoclonal antibody (Pathfinder Chlamydia Confirmation System; Bio-Rad, Redmond, WA) and counterstained with Evan's blue. Images were captured in 10 random fields with a fluorescence microscope (Leica DMLB, Germany) with standard filters at $40 \mathrm{X}$ magnification. The inclusions were counted as described under section Immunofluorescence microscopy. Data from 3 independent experiments were combined to calculate the mean and standard deviation.

\section{Gene expression analysis by real-time PCR}

For the analysis of chlamydial gene expression, infected cells were harvested at different time points and realtime PCR was performed targeting the 16S rRNA gene as described previously [34]. To analyse chlamydial developmental phase, expression of genes eиo, отрA and $o m c B$ were performed. All the primers were ordered from Eurofins MWG Operon (Additional file 1: Figure S1). Briefly, total RNA was isolated from the cell pellets of infected monocytes or DCs using the Macherey Nagel kit (Macherey-Nagel GmbH, Dueren, Germany). 500 nanogram of RNA was reverse-transcribed from each sample using the Eurogentec Reverse-Transcription Kit. Real-time PCR was performed using the qPCR Core kit (Eurogentec) in Roche Lightcycler 480 system. The gene expression levels were calculated by the delta-delta $\mathrm{Ct}(\mathrm{ddCt})$ method [41], normalized to $16 \mathrm{~S}$ in case of chlamydial genes and to $18 \mathrm{~S}$ for host genes, and compared to the mock sample as the reference gene. The specificity and identity of the amplified products were determined using Light Cycler 480 melting curve analysis software. Data from 3 independent experiments with pool of 2 donors were combined to calculate the mean and standard deviation. 


\section{Quantification of cytokines}

The level of the cytokines IL-1 $\beta$, IL-6, IL-8, IL-10, TNF and IL-12p70 were measured in the supernatants of the infected monocytes and DCs collected 1 day p.i. by Cytometric Bead Array (Human Inflammatory Cytokines Kit; BD Biosciences, San Diego, CA) according to the manufacturer's instruction. In brief, $50 \mu \mathrm{L}$ of human inflammation capture bead suspension and $50 \mu \mathrm{L}$ of phycoerythrin detection reagent were added to an equal amount of samples or standard dilution and incubated for 3 hours at room temperature in the dark. The monocyte samples were diluted 1:2 and DCs samples were diluted 1:4 with assay diluent to have sample data within the range of the standard curve. Subsequently, samples were washed with wash buffer and centrifuged at $200 \times g$ at room temperature for 5 minutes. The samples were further fixed with $2 \%$ paraformaldehyde for 30 minutes. The supernatant was discarded and $300 \mu \mathrm{L}$ of wash buffer was added. Samples were then analysed on a BD FACS Calibur flow cytometer (BD Biosciences, Heidelberg, Germany). The data was analyzed using the FCAP array software (BD Biosciences). Data from 3 independent experiments with pool of 2 donors were combined to calculate the mean and standard deviation.

\section{Innate and adaptive immune response array}

The Human Innate and Adaptive Immune response Array (PAHS-052) was performed using the SYBR green-based RT2 Profiler system (SA Biosciences, Frederick, MD). This PCR array is a pathway focused array that contains a set of 84 related genes involved in the inflammatory immune response. This assay also contains 5 housekeeping genes and 3 other reaction controls to assess genomic DNA contamination, RNA quality, and general PCR performance. Total RNA from infected monocytes and DCs were extracted using Macherey Nagel kit (Macherey-Nagel GmbH, Dueren, Germany). Due to the low RNA concentration, monocyte RNA sample were amplified by $\mathrm{RT}^{2}$ PreAmp PCR master mix (SA Biosciences, Frederick, MD). Equal amount of RNA from each sample was reverse-transcribed to cDNA by using Reverse-transcription mix preceded by a genomic DNA elimination step; both provided in the kit. The amplification of the immune genes in the samples was investigated by real-time PCR on Roche Light Cycler 480 according to the manufacturer's instructions. Data analysis was performed using manufacturer's program and is based on the ddCt method, with normalization of the raw data to the panel of housekeeping genes provided in the array. The genes showing modulation by 1.5 fold up or down were only selected for further analysis. Functional annotations of the selected genes were carried out by the bioinformatics software David for Bioinformatics. Three independent experiments with a pool of 2 donors each were analyzed.

\section{Statistical analysis}

Statistical evaluation of the data was done using GraphPad Prism 5 software. Student t-test was performed for simple comparison between 2 means. For multiple comparisons, the results were analysed by two-way ANOVA followed by Bonferoni's post-test. $\mathrm{p}<0.05$ was considered statistically significant. All shown data are representative for at least 3 independent experiments.

\section{Results}

Chlamydia trachomatis infect monocytes and monocyte-derived DCs in a comparable manner

Monocytes isolated from human peripheral blood mononuclear cells (PBMCs) and monocyte-derived DCs were infected with $C$. trachomatis serovars $\mathrm{Ba}, \mathrm{D}$ and $\mathrm{L} 2$ (Figure 1). Results show that all the three serovars were capable of infecting both the monocytes and DCs and form inclusions as detected by immunofluorescence microscopy 2 days post infection (p.i.). However, the inclusions were smaller in size compared to typical inclusions that have been reported in HeLa cells (Additional file 2: Figure S2). The inclusion morphology and staining intensity varied between the infected monocytes and DCs.

In monocytes, the percentage of infected cells were comparable among the three serovars and did not seem to change even when the infection duration was extended to 3 days (Table 1). For DCs, the percentage of infected cells were similar for serovars $\mathrm{Ba}$ and $\mathrm{D}$ but serovar L2 showed a higher infection rate as compared to the other two (Table 1). However, the infection rate declined remarkably for all the three serovars when infected for 3 days. The infection rate was nevertheless much lower in both monocytes and DCs than in HeLa. Mock controls were prepared for each round of experiments which showed absence of chlamydial antigens in the donors (Additional file 3: Figure S3).

\section{Differential development of $C$. trachomatis serovar $L 2$ in monocytes and DCs}

In our study, we further investigated the survival and re-infection potential of chlamydia serovars after the primary infection of monocytes and DCs. Chlamydiainfected monocytes and DCs were harvested 2 days p.i. and passaged onto HeLa cell confluent monolayer. HeLa cells were investigated by immunofluorescence microscopy 2 days p.i. and the inclusions were counted.

The serovars $\mathrm{Ba}$ and the $\mathrm{D}$ were not able to produce inclusions in HeLa cells after infecting either monocytes or DCs for 2 days. Only scattered antigens could be detected (Figure 2). Interestingly, serovar L2 produced inclusions in HeLa cells after infecting both monocytes and DCs (Figure 2). There was no recovery of infectious progeny from serovars $\mathrm{Ba}$ and $\mathrm{D}$ even with longer 

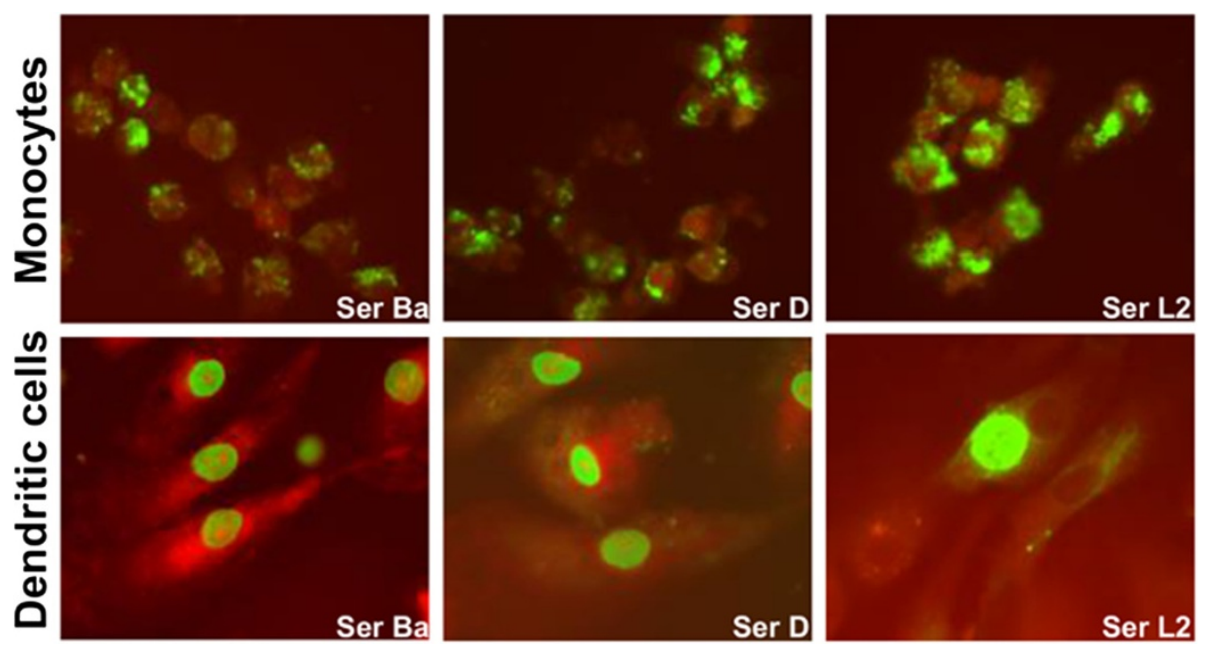

Figure $1 \mathrm{Immunofluorescence} \mathrm{microscopy} \mathrm{of} \mathrm{infected} \mathrm{monocytes} \mathrm{and} \mathrm{monocyte-derived} \mathrm{Dendritic} \mathrm{cells} \mathrm{(DCs).} \mathrm{Monocytes} \mathrm{(upper} \mathrm{panel)}$ and monocyte-derived DCs (lower panel) were infected with C. trachomatis serovars Ba, D and L2 (MOI-3) for 2 days. Chlamydial inclusions (green) were stained with FITC conjugated anti-chlamydia LPS antibody and counterstained with Evans Blue. Pictures were taken at 63X magnification with Leica DMLB. The figures are representative of 3 independent experiments.

duration of primary infection (3 days) or if the passage in HeLa cells was carried out for a longer duration (72 hours) (data not shown). In the case of serovar L2, passaging for longer time did not yield a higher number of infectious progeny.

\section{Metabolic activity of chlamydia within infected monocytes and DCs}

To characterize the metabolic activity of chlamydiae in monocytes and DCs, we investigated the expression of 16S rRNA gene transcripts which reflects the growth rate and/or metabolic activity of chlamydiae in the cells [40]. The expression of $16 \mathrm{~S}$ rRNA in chlamydiaeinfected monocytes and DCs was assessed over 3 days after infection.

$16 \mathrm{~S}$ rRNA was highly expressed in the infected monocytes for all three chlamydia serovars $\mathrm{Ba}, \mathrm{D}$ and $\mathrm{L} 2$

\section{Table 1 Comparison of infection rate in monocytes and monocyte-derived DCs infected with $C$. trachomatis serovars $\mathrm{Ba}, \mathrm{D}$ and $\mathrm{L} 2$}

\begin{tabular}{|c|c|c|c|c|c|c|}
\hline \multicolumn{7}{|c|}{ Infection percentage (\%) } \\
\hline & \multicolumn{2}{|c|}{ Serovar Ba } & \multicolumn{2}{|c|}{ Serovar D } & \multicolumn{2}{|c|}{ Serovar L2 } \\
\hline & $\begin{array}{l}2 \text { days } \\
\text { p.i }\end{array}$ & $\begin{array}{l}3 \text { days } \\
\text { p.i }\end{array}$ & $\begin{array}{l}2 \text { days } \\
\text { p.i }\end{array}$ & $\begin{array}{l}3 \text { days } \\
\text { p.i }\end{array}$ & $\begin{array}{l}2 \text { days } \\
\text { p.i }\end{array}$ & $\begin{array}{l}3 \text { days } \\
\text { p.i }\end{array}$ \\
\hline Monocytes & $32 \pm 5$ & $34.3 \pm 6$ & $33.6 \pm 6$ & $36.6 \pm 7$ & $44 \pm 6$ & $42 \pm 3$ \\
\hline$x$ & $26.8 \pm 2$ & $20.7 \pm 2$ & $29.4 \pm 1$ & $24.4 \pm 1$ & $39.9 \pm 4$ & $25.4 \pm 2$ \\
\hline leLa & $78 \pm 7$ & $81.3 \pm 6$ & $83.5 \pm 4$ & $85.1 \pm 7$ & $88.7 \pm 3$ & $84.2 \pm 3$ \\
\hline
\end{tabular}

Monocytes, DCs and HeLa cells were infected with Chlamydia trachomatis serovars $\mathrm{Ba}, \mathrm{D}$ and $\mathrm{L} 2$ and stained with anti-Chlamydia LPS antibody at 2 day and 3 day p.i.. Quantification of chlamydia infected cells were done by counting total number of cells (indicated by nuclei staining) and cells positive for Chlamydia and from 15 pictures The mean and \pm SD were calculated from three independent experiments. throughout the 3 day time course of infection (Figure 3). The heightened metabolic activity of the intracellular chlamydia did not seem to decline with a longer duration, thus indicating that all the serovars were viable and metabolically active within the monocytes.

In contrast $16 \mathrm{~S}$ rRNA expression level was negligible in DCs for serovars Ba and D at 1 day p.i. and further declined with infection progression (Figure 3). Serovar L2 displayed highly significant expression of 16S rRNA at 1 and 2 day p.i. Although the level declined on the 3 day p.i., the expression remained significant (Figure 3).

To further characterize developmental state of chlamydial serovars within the infected monocytes and DCs, gene expression of euo, ompA and $о m c B$ were investigated. Each of these genes are known to be expressed at different developmental stages of chlamydiae (early, mid and late phase respectively), and have previously reported to be transcriptionally altered during chlamydial growth in human monocytes and DCs [40,42]. Figure 4 depicts the expression of the three genes in monocytes and DCs respectively. Expression of the 3 genes within serovars $\mathrm{Ba}$ and $\mathrm{D}$ in both cell types was similar and stable, albeit at low levels in all the three time points that were investigated. Serovar L2 depicted a different pattern; early stage gene euo was significantly expressed 1 day p.i. compared to serovars $\mathrm{Ba}$ and $\mathrm{D}$, gradually diminishing with time in both monocytes and DCs. The expression of mid-cycle gene ompA for serovar L2, although higher than the serovars $\mathrm{Ba}$ and $\mathrm{D}$, was not statistically significant in infected monocytes. The expression for ompA within infected DCs peaked at 2 day p.i. significant to both serovars $\mathrm{Ba}$ and $\mathrm{D}$. Expression of late stage gene $o m c B$ increased significantly 3 days p.i. for serovar 

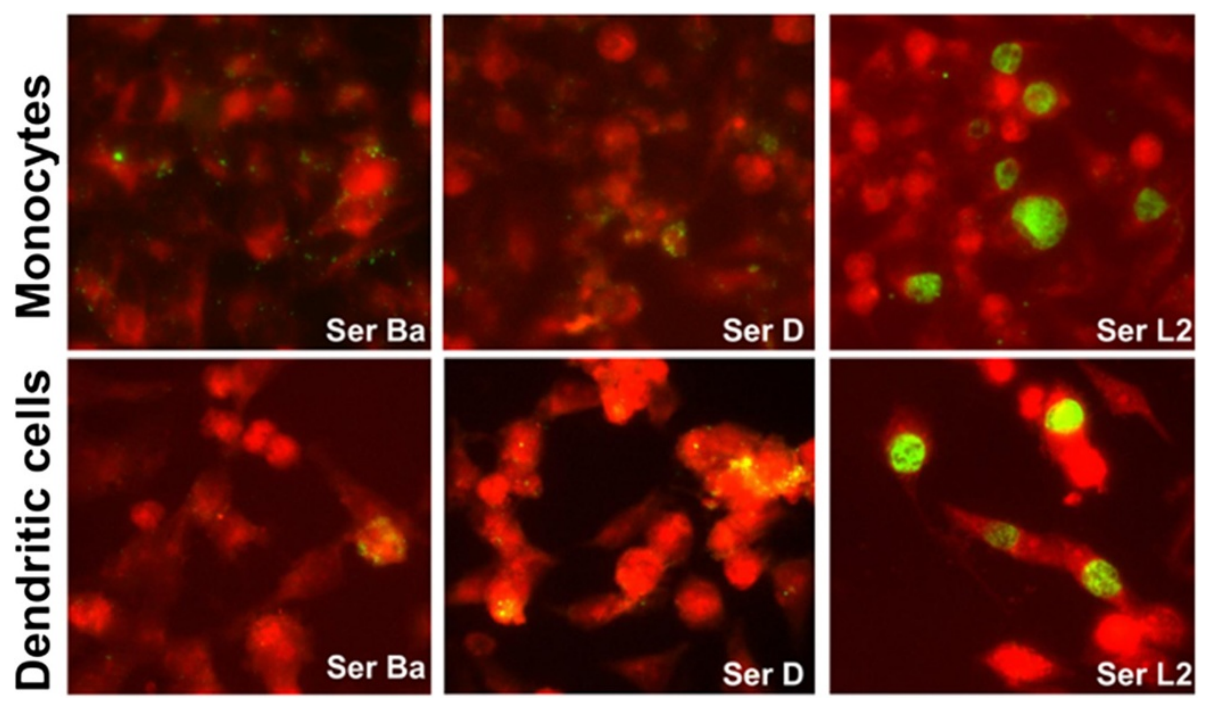

Figure 2 Infectivity assay of Chlamydiae infected monocytes and monocyte-derived DCs. Monocytes (upper panel) and human monocyte-derived DCs (lower panel) were infected with C. trachomatis serovars Ba, D and L2 (MOI-3) for 2 days and were further passaged in HeLa cells for 2 days. Chlamydial inclusions (green) were stained with FITC conjugated anti-chlamydia LPS antibody and counterstained with Evans Blue. Pictures taken at 40X magnification with Leica DMLB. The figures are representative of 3 independent experiments.

L2 compared to serovars $\mathrm{Ba}$ and $\mathrm{D}$ in both monocytes and DCs.

Indolamine 2, 3-dioxygenase expression is down-regulated in monocytes and upregulated in DCs by $C$. trachomatis serovar L2

One aspect of chlamydial infection is the gamma-interferon (IFN- $\gamma$ ) mediated induction of Indolamine-2, 3-dioxygenase (IDO), an enzyme catabolizing breakdown of tryptophan in culture media. The unavailability of this essential amino acid can lead to chlamydial growth arrest termed as persistence [43]. The role of TNF- $\alpha$ mediated IDO induction in DCs [44] as well IFN- $\gamma$ independent IDO activation in monocytic THP-1 cells have been reported earlier [45]. We considered that the level of IDO gene expression could be crucial in understanding the contrasting infection outcome by the chlamydia serovars in monocytes and monocyte-derived DCs. Hence the expression of IDO gene in chlamydiae-infected monocytes and DCs was detected over 3 days post infection.

Monocytes, infected with serovars $\mathrm{Ba}$ and $\mathrm{D}$ expressed higher levels of IDO 1 day post infection (Figure 5). Contrastingly, IDO expression by serovar L2 infected monocytes was significantly down-regulated 1 day p.i compared to serovar D. On the other days of infection the trend was similar but not significant.

IDO expression was significantly up-regulated in DCs infected with serovar L2 (Figure 5) compared to serovars $\mathrm{Ba}$ and $\mathrm{D}$. IDO expression declined throughout the infection course for all the servers, however maintaining a significant expression for serovar L2 infection.
Attempts were made to enhance chlamydial recovery from infected monocytes and DCs by addition of Tryptophan, known to be depleted by IDO during chlamydial infection $[34,46]$. However the infected cultures supplemented with Tryptophan $(200 \mu \mathrm{g} / \mathrm{ml})$ when passaged on HeLa cells could not abrogate the growth arrest; chlamydial inclusions could not be recovered from serovar $\mathrm{Ba}$ and D cultures (data not shown). However, Serovar L2 could produce chlamydial inclusions irrespective of Tryptophan.

\section{Differential cytokine response induced in monocytes and DCs by chlamydial infection}

We investigated the role of cytokines in mediating contrasting infection outcome of chlamydia infection the monocytes and DCs. Supernatants were collected from monocyte and monocyte-derived DCs culture infected with C. trachomatis serovars Ba, D and L2 at 1 day p.i. and cytokine responses were assessed by Cytokine Bead Array.

As depicted in Figure 6, infection of monocytes and monocyte-derived DCs with chlamydial serovars Ba, D and L2 could induce detectable secretion of cytokines IL-8, IL-6, IL-1 $\beta$, IL-10 and TNF. Production of IL12 p70 was below the standards (data not shown).

Pro-inflammatory cytokines IL-1 $\beta$ and TNF was elevated in the chlamydiae infected monocytes than the mock control, however were not statistically significant. The level of cytokines IL-6 and IL-8 in infected monocytes showed no statistical difference with mock control. The anti-inflammatory cytokine IL-10 was induced in 


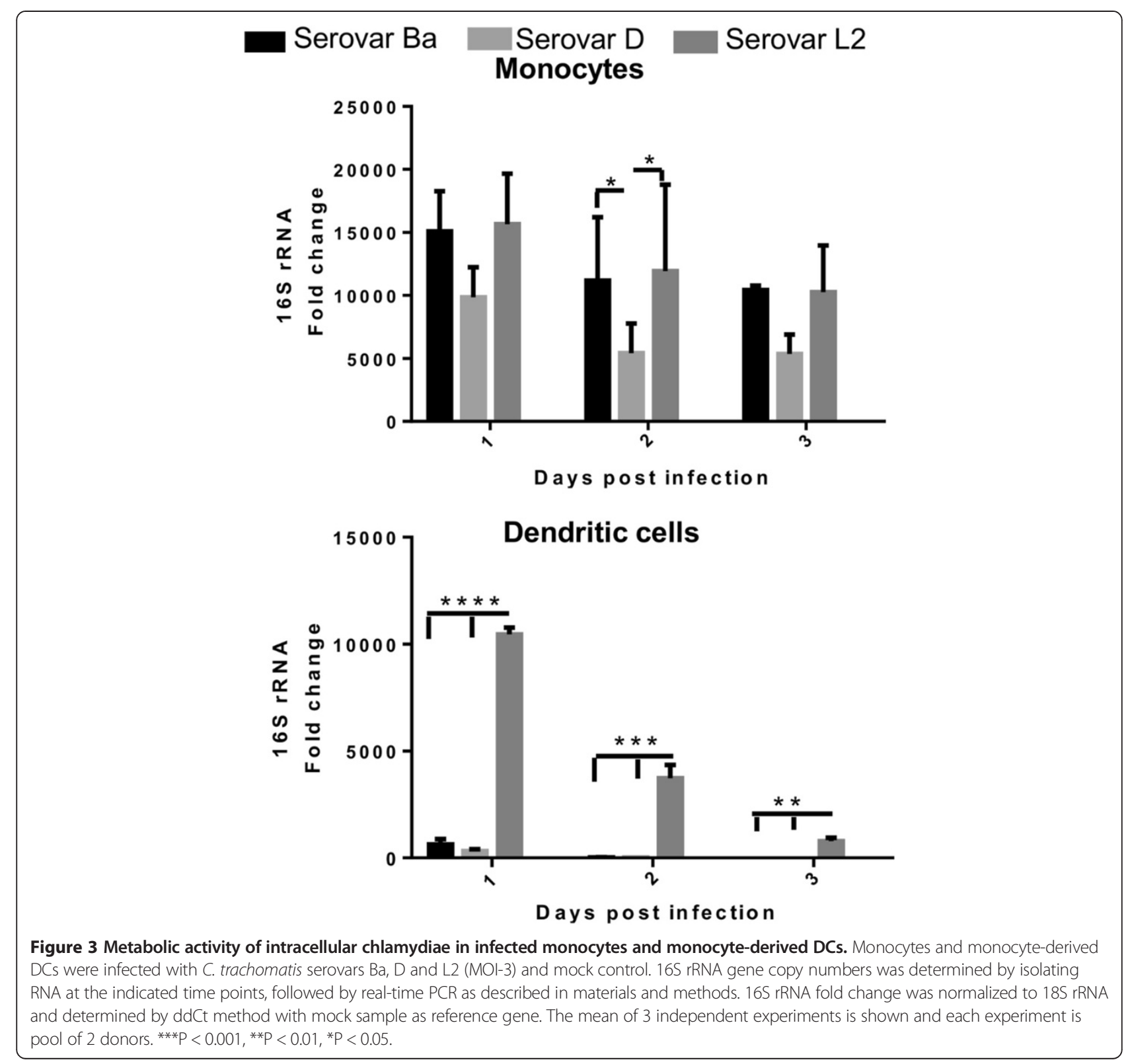

higher levels than the mock with serovar $\mathrm{Ba}$ infection secreting significant amounts compared to mock. DCs infected with serovars D and L2 showed significantly up-regulated levels of TNF. The other pro-inflammatory cytokine IL-1 $\beta$ although secreted in higher amounts within serovar L2 infected DCs, than the other serovars or mock, was not significant. DCs infection resulted in significant production of inflammatory cytokines IL-8 and IL-6. The anti-inflammatory cytokine IL-10 levels were low in the infected DCs and were not statistically significant to the mock control. To understand LPS contribution in the observed cytokine responses, monocytes and DCs were infected with heat-killed C. trachomatis serovars Ba, D and L2 EBs at MOI-3 and the cytokine levels were investigated (Additional file 4: Figure S4). Heat-killed EBs for serovar Ba and D induced significantly low level of IL8 and IL-6 in monocytes while the TNF levels were low in DCs for serovar D and L2. The most remarkable observation was the negligible induction of IL-10 by heat-killed EBs from all 3 serovars in monocytes which was highly significant.

\section{Immune gene response to $C$. trachomatis infected monocytes and DCs}

To determine the host genes activated by chlamydia infection, the immune response was analyzed by Human innate and Adaptive Immune response array. Genes differentially regulated 1.5 fold up or down in monocytes 




Figure 4 Quantification of euo, ompA and omcB gene expression in chlamydiae infected monocytes and monocyte-derived DCs. Monocytes and monocyte-derived DCs were infected with C. trachomatis serovars Ba, D and L2 (MOl-3) and mock control. Copy numbers of euo, OMPA and $O M C B$ genes were determined by isolating RNA at the indicated time points, followed by real-time PCR as described in materials and methods. Gene fold change was normalized to chlamydial $16 \mathrm{~S}$ rRNA and determined by ddCt method with mock sample as reference gene. The mean of 3 independent experiments is shown and each experiment is pool of 2 donors. ${ }^{* *} P<0.001,{ }^{* *} P<0.01,{ }^{*} P<0.05$.

or monocyte-derived DCs infected with C. trachomatis serovars Ba, D and L2 24 hours p.i. were considered for further analysis (Figure 7).

As depicted in Figure 7, Serovar Ba induced up regulation of 11 genes, Serovar D of 11 genes and serovar L2 of 13 genes within infected monocytes. Of these up-regulated genes 8 genes were common in all 3 serovars which included receptor for bacterial components (PGLYRP3) and genes responsible for antibacterial defense (DEF4BA, CCL2). Cytokine genes inducing antiviral effect (IFNA1, IFNB1) as well as immune-regulation (IL-10) were also elevated emphasizing the cytokine interplay in infected monocyte. It is noteworthy that Toll-like receptor (TLR) 3 which recognizes dsRNA and is crucial for the TRIF mediated immune response pathway (MyD88 independent) was up-regulated. TREM1 gene, which is an important sepsis marker, was elevated in serovar L2 infected monocytes.

The down-regulated genes in the infected monocytes numbered 19 for serovar Ba, 15 for serovar D and 14 for serovar L2 (Figure 7). Ten of those genes were common for all the 3 serovars which included a member of Myd88 dependent pathway (TLR8) and interacting protein (TOLLIP). Other genes involved were predominantly involved in vascular mechanism (PTAFR, PPBP, FN1 and COLEC12). Additionally, some genes involved in apoptosis and oxidative process (CHUK, NCF4 and NLRC4) were also down-regulated.

DCs response to the chlamydial serovars were also intriguing. There was up regulation of 4 genes by serovar $\mathrm{Ba}, 7$ genes by serovar $\mathrm{D}$ and 10 genes by serovar L2 (Figure 7). The remarkable observation was that serovars $\mathrm{Ba}, \mathrm{D}$ and L2 could all up regulate TLR8 as well other TLRs individually (TLR, 2, 4 and 6), all belonging to the Myd88 dependent signalling pathway [47].

The genes down regulated in DCs in response to chlamydial infection numbered 4 for serovar Ba, 5 for serovar D and 5 for serovar L2. Two genes were common which included anti-inflammatory effector (IL-10) as well as gene involved in vascular process (COLEC12). 


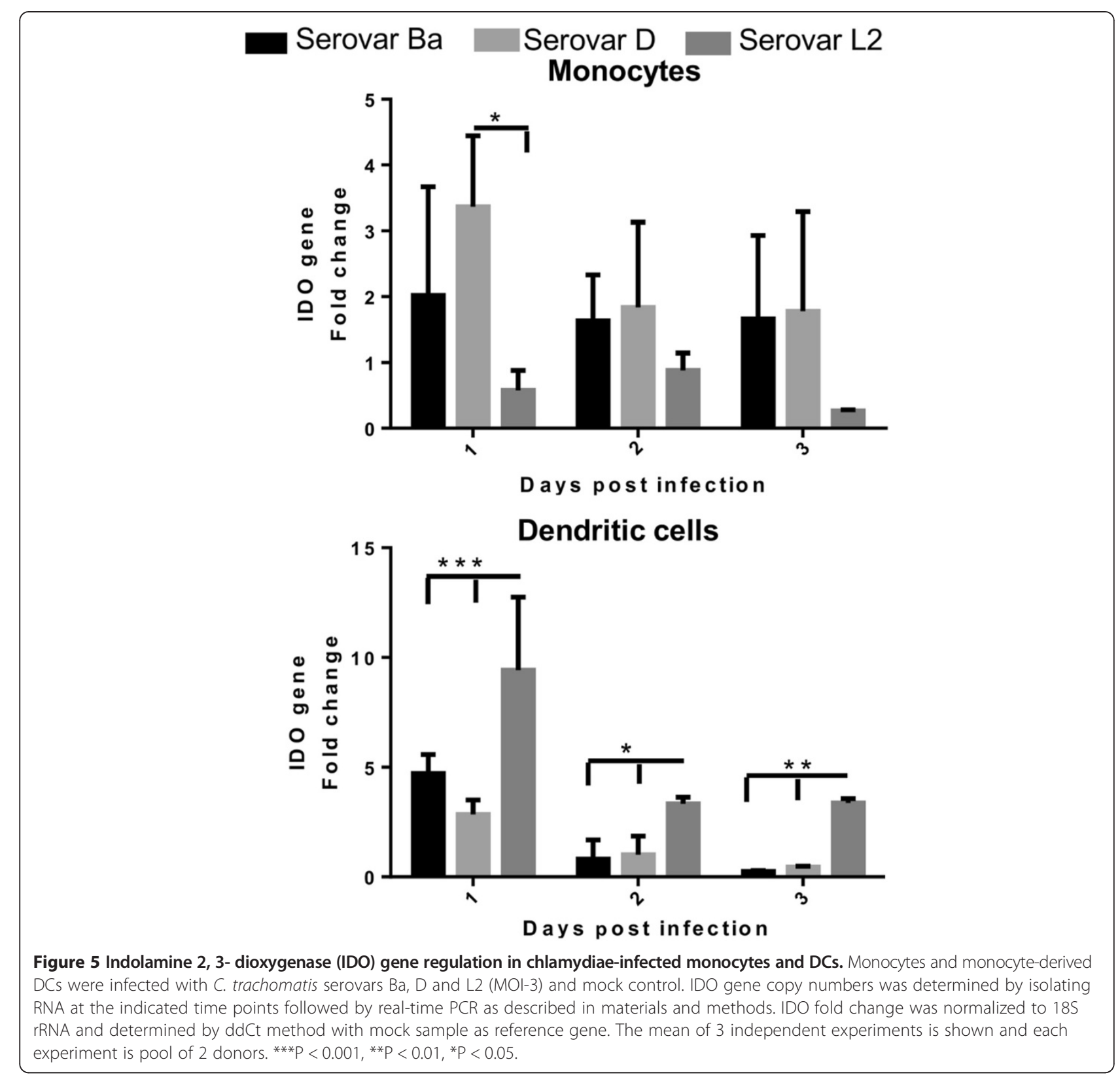

\section{Discussion}

In our study we could demonstrate that the different serovars of $C$. trachomatis experience altered fate in monocytes and DCs by virtue of the variable host immune response induced by infection. Monocytes and DCs could be primarily infected by $C$. trachomatis serovars $\mathrm{Ba}, \mathrm{D}$ and $\mathrm{L} 2$ in comparable degree. This is in agreement with previous study showing similar results in terms of primary infection of DCs by C. trachomatis [31]. To our knowledge, no such study has been reported for monocytes, hence we report here for the first time characteristics of $C$. trachomatis serovars $\mathrm{Ba}, \mathrm{D}$ and L2 infection in monocytes. The infection percentages were comparable for serovar $\mathrm{Ba}$ and $\mathrm{D}$ while serovar L2 experienced a slightly higher rate in both monocytes and DCs infection. Incidentally the percentage declined in infected DCs with longer culture period for all the three serovars. The variable inclusion morphology and lower infection rate within chlamydiae-infected monocytes and DCs as opposed to in HeLa indicated that replication cycle of C. trachomatis was somewhat restricted in these two immune cell types.

Attempts to recover infectious particles from the monocytes and DCs infected with the chlamydia serovars led to an interesting observation. Monocytes infected with serovars $\mathrm{Ba}$ and $\mathrm{D}$ when passaged to HeLa cells could not produce any inclusions, which is in accordance with earlier studies demonstrating inability of 



Figure 6 Cytokine concentration in chlamydiae-infected monocytes and monocyte-derived DCs. Monocytes and monocyte-derived DCs were infected with C. trachomatis serovars Ba, D and L2 (MOl-3) and mock control. Supernatants were collected 1 day post infection and the concentration of the different cytokines IL-1 $\beta, T N F, I L-6, I L-8$ and IL-10 were determined by using the kit Cytometric Bead Array. The concentration is reported as $\mathrm{pg} / \mathrm{ml}$. The cytokine secreted by heat-killed sample of each serovar were quantified and are indicated for each dataset. The mean of 3 independent experiments is shown and each experiment is pool of 2 donors. ${ }^{* *} \mathrm{P}<0.001,{ }^{* * P}<0.01,{ }^{*} \mathrm{P}<0.05$.

serovar $\mathrm{K}$ to productively infect monocytes [23,24]. In contrast to earlier observations [48] where mononuclear cells were considered to be microbicidal for all C. trachomatis serovars our results revealed that serovar L2 could productively infect monocytes.

A similar trend was observed in DCs, where serovar $\mathrm{Ba}$ and serovar D showed abortive infection with no typical inclusions. Infectious particles could only be recovered from serovar L2 infected DCs as has been reported previously by Gervassi et al. [31]. However Gervassi et al. showed that serovar E passaged in DCs could be further propagated in HeLa cell whereas in our study serovar D, member of the same biovariant could not be propagated in HeLa cells. The differences between these findings can be caused by effects of genetic human host polymorphism (source of DCs are different), differences in culture condition (10\% FCS vs 10\% autologous serum), or use of different MOIs 10 vs 3 ).

In conjunction with the reinfection data, high expression levels of $16 \mathrm{~S}$ rRNA within monocytes infected with serovars $\mathrm{Ba}, \mathrm{D}$ and $\mathrm{L} 2$ indicated that $C$. trachomatis serovars were viable throughout the infection period, even though infectious progeny could only be recovered from L2 infected monocytes. This phenomenon of viability without producing infectious bodies is known as chlamydial persistence $[18,49]$. Serovar K infection of monocytes resulted in attenuation of new EB production although genes involved in chlamydial DNA replication were expressed during persistence [20]. Nevertheless our study establishes that this is a general phenomenon occurring in monocytes for several serovars of $C$. trachomatis biovariants. Contrasting observations are provided by DCs infected with the chlamydial serovars. The absence of recovered infectious progeny along with the negligible expression of $16 \mathrm{~S}$ rRNA in serovars Ba and D in infected DCs 2 days p.i. suggest the loss of metabolic activity of $C$. trachomatis serovars within DCs. This loss of metabolic activity of $C$. trachomatis serovars within DCs indicated towards a probable degradation of chlamydiae. Serovar L2 could however, produce inclusions 


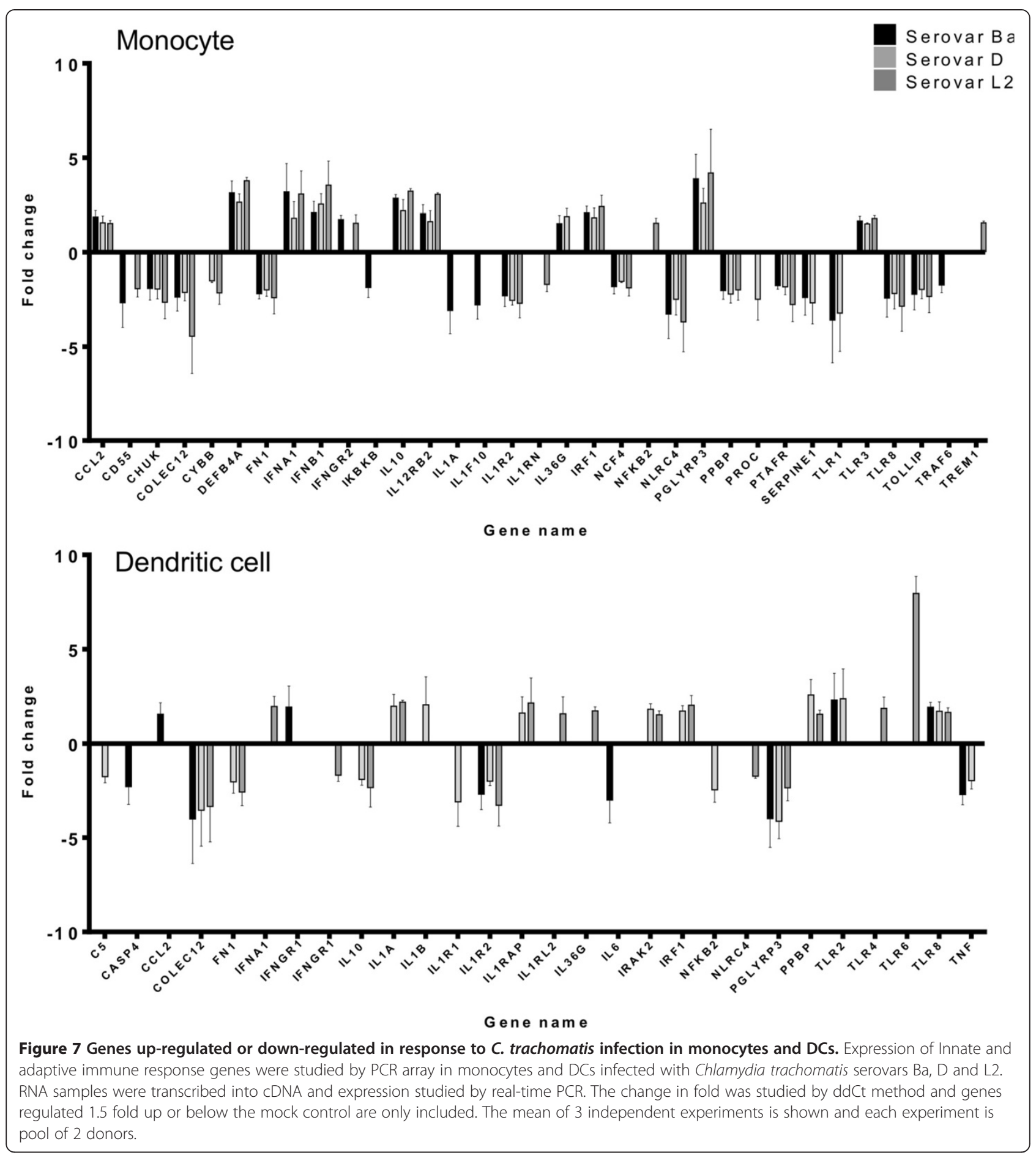

during reinfection studies and express 16S rRNA 2 days p.i. in DCs but suffered rapid decline in viability 3 days p.i.. DCs have shown the ability to degrade Chlamydia psittaci and C. trachomatis MoPn [29] but other DC- C. trachomatis studies have shown contrasting results, where they showed that $C$. trachomatis serovars were confined within specific vacuoles within DCs being able to replicate $[30,31]$. Our results were in contrast to Chlamydia pneumoniae infected DCs showing an increase in $16 \mathrm{~S}$ rRNA expression when infected for 3 days [34]. The study of the chlamydial developmental cycle within the monocytes and DCs by expression of stage-specific genes showed a clear prominence of serovar L2 compared to serovars $\mathrm{Ba}$ and $\mathrm{D}$. The observed gene expression for serovar L2 was in accordance with the expected early, mid and late phase patterns and therefore indicative of presence of 
viable chlamydiae. The difference in gene expression between serovar L2 and the serovars $\mathrm{Ba}$ and D indicates the infection severity. The expression of ompA and $о m c B$ genes for serovars $\mathrm{Ba}$ and $\mathrm{D}$, within monocytes and $\mathrm{DCs}$, at later time points indicate that some chlamydiae were still viable. While in monocytes these chlamydia were in persistent form, it is possible that in DCs transient level of C. trachomatis development is allowed while predominantly inhibiting or degrading the pathogen as it has been reported previously for other monocytic cells [50].

The presence of a functional tryptophan synthase gene in urogenital serovars and the absence of it in ocular serovars has been related with tissue tropism [35,37]. The tryptophan synthase gene enables the bacteria to use indole as a substrate for tryptophan synthesis when the intracellular tryptophan is depleted by IDO induction during chlamydial infection. In this study, we have shown that IDO expression levels for ocular serovar Ba and urogenital serovar D were similar while LGV serovar L2 showed down-regulation in infected monocytes. In infected DCs, IDO expression was significantly upregulated for serovar L2 but declined rapidly in the other two serovars. The involvement of TNF secreted by DCs (Figure 6) seemed to be crucial in the up-regulation of IDO, as TNF has been earlier reported to activate IDO expression in human DCs [51]. The heightened level of IDO in serovar L2 could not restrict its active infection probably due to the presence of functional tryptophan synthase in genital serovars as discussed above. IDO expression revealed analogous pattern for serovars $\mathrm{Ba}$ and $\mathrm{D}$ in both monocytes and DCs which poses a query whether the organotropism is less pronounced within the immune cells.

In infected monocytes the pro-inflammatory cytokines TNF and IL-1 $\beta$ were secreted in higher levels than mock which might be the reason for the restricted chlamydial growth observed, higher secretion of these cytokines has also been reported previously [45]. The significance of TNF in serovar D and L2 infected DCs confirmed their role in restricting chlamydial growth. The inflammatory cytokines IL-8 and IL-6 although secreted in higher levels by the infected monocytes were not significant. One probable reason for such high volumes could be due to the monocyte adherence for 2 hours prior infection which might have induced some activation. The fact that IL-10 was highly induced by serovars Ba, D and L2 within monocytes demonstrates the critical role played by the anti-inflammatory process to prevent degradation of chlamydia and remain viable within the monocytes. DC infection with serovars Ba, D and L2 could induce significant levels of inflammatory cytokines IL-6 and IL8. The anti-inflammatory IL-10 was secreted in low levels by the serovars, thus displaying dominance of the inflammatory process in DC infection. The distinct interplay of pro-inflammatory and anti-inflammatory cytokines seemed to play role in infection outcome within monocytes and DCs. The cytokine studies with heatkilled EBs showed that TNF was induced by active infection of DCs by serovars D and L2. Infection by viable chlamydia could only induce secretion of IL-10 in monocytes, indicating that an active infection is essential for inducing these particular cytokines in monocytes or DCs. The data demonstrated that monocytes and DCs induce altered levels of cytokines in response to chlamydial infection, and DCs demonstrate a stronger inflammatory role than the monocytes.

Our data manifested distinct activation profiles of immune genes in monocytes and DCs during $C$. trachomatis infection. Although, the fold-regulation was not significant, the differential regulation of the different genes when analysed through functional annotation tool, David for Bioinformatics, could reveal an interesting pattern. The hallmark of this response was the involvement of the Toll like receptor (TLR) signalling pathway-critical mediators of innate immune response recognizing different microbial components [52-54]. On contact with their ligands, TLRs engage different adapter molecules to propagate the downstream signalling. The adapter molecule MyD88 is used by all the TLRs (except TLR3) to activate the transcriptional activator NF- $\mathrm{KB}$ and induce secretion of TNF, IL-6 and other inflammatory cytokines thus forming the MyD88 dependent pathway $[47,55]$. The other pathway recruits TRIF adapter molecule to induce IFN $\beta$ and late induction of NF- $\mathrm{KB}$ constituting the MyD88 independent pathway $[47,56]$. TLR3 is able to signal exclusively through MyD88-independent pathway [57]. The involvement of TLR2 and TLR4 in C. trachomatis mediated infection response has been reported by earlier studies $[58,59]$. In our studies the up-regulation of TLR3, IFNA1, IFNB1 for serovars $\mathrm{Ba}, \mathrm{D}$ and $\mathrm{L} 2$ in infected monocytes and the simultaneous down regulation of TLR1, TLR8 suggests the dominance of the TRIF mediated signalling in C. trachomatis infected monocytes. The converse could be seen in C. trachomatis infected DCs where TLR8 was upregulated for all the serovars and TLR/2/4/6 of MyD88 signalling pathway were differentially up-regulated for the different serovars. With the array findings, one could speculate that two distinct immune response pathways are employed by monocytes and DCs when infected with specific chlamydial serovars. The results could be further studied in detail to ascertain the role played by the different adapter mediated signalling pathways in chlamydia infection.

\section{Conclusion}

Our study demonstrated that C. trachomatis serovars Ba, D and L2 infected monocytes and DCs in a comparable manner; however, they underwent differential infection 
consequences. Serovars $\mathrm{Ba}$ and $\mathrm{D}$ became persistent in monocytes while they degraded within DCs. Serovar L2 could, however, maintain the development cycle in both monocytes and DCs, although the process was severely impaired. The heightened levels of inflammatory cytokines secreted by the chlamydial infection in DCs compared to monocytes could be instrumental to the differences observed. The host immune genes response to infection displayed distinct activation profile within monocytes and DCs. Collectively, we could establish that the host pathogen interaction in chlamydia infection is not only serovar specific but also cell specific.

\section{Additional files}

Additional file 1: Figure S1. Gene specific primers used for quantitative real-time PCR.

Additional file 2: Figure S2. Immunofluorescence microscopy of HeLa cells: HeLa cells were infected with $C$. trachomatis serovars $\mathrm{Ba}, \mathrm{D}$ and $\mathrm{L} 2$ (MOl-3) for 2 days as positive control. Chlamydial inclusions (green) were stained with FITC conjugated anti-chlamydia LPS antibody and counterstained with Evans Blue. Pictures were taken at 40X magnification with Leica DMLB. The figures are representative of 3 independent experiments.

Additional file 3: Figure S3. Immunofluorescence microscopy of mock-infected monocytes and monocyte-derived DCs: Monocytes and monocyte-derived DCs were infected with mock control for 2 days. Chlamydial inclusions (green) were stained with FITC conjugated antichlamydia LPS antibody and counterstained with Evans Blue. Pictures were taken at 40X magnification with Leica DMLB. The figures are representative of 3 independent experiments.

Additional file 4: Figure S4. Effect of heat-killed chlamydia in cytokine induction within infected monocytes and monocyte-derived DCs: Monocytes and monocyte-derived DCs were infected with live and heat-killed EBs of C. trachomatis serovars $\mathrm{Ba}, \mathrm{D}$ and $\mathrm{L} 2(\mathrm{MOl}-3)$ and mock control. Supernatants were collected 1 day post infection and the concentration of the different cytokines IL-1 $\beta, T N F, I L-6, I L-8$ and IL-10 were determined by using the kit Cytometric Bead Array. The concentration is reported as pg/ml. The mean of 3 independent experiments is shown and each experiment is pool of 2 donors. ${ }^{* *} P<0.001$, ${ }^{* *} P<0.01$, ${ }^{*} P<0.05$.

\section{Abbreviations}

C.trachomatis: Chlamydia trachomatis; EB: Elementary body; DC: Dendritic cell; MOI: Multiplicity of infection; p.i.: Post infection; IL: Interlukein.

\section{Competing interests}

The authors declare that they have no competing interests.

\section{Authors' contribution}

BD performed the experiments, acquired, analyzed and interpreted the data, and drafted the manuscript. FN and ADW: made substantial contributions to the conception and design of experiments, interpretation of results, and drafted and critically revised the manuscript. JT and $\mathrm{HH}$ made substantial contributions to the conception and design of experiments. All authors read and approved the final manuscript.

\section{Acknowledgements}

This work was supported by the Hannover Biomedical Research School (HBRS) and the Center for Infection Biology (ZIB). We appreciate the invaluable assistance of Dr Thorsten Volgmann for providing us with buffy coats. We are grateful to Barbara Hertel for her technical assistance, Anna Buch for microscopy assistance and Jenny Bode for her critical reading and correction of the manuscript.
Received: 10 February 2014 Accepted: 21 July 2014

Published: 14 August 2014

\section{References}

1. Wang SP, Grayston JT: Three new serovars of Chlamydia trachomatis: Da, la, and L2a. J Infect Dis 1991, 163:403-405.

2. World Health Organization: Making progress toward the global elimination of blinding trachoma. Geneva: Report 10th Meet WHO Alliance Glob Elimin Blind Trach; 2006.

3. Bryan CP: trachoma origin. London: papyrus Ebers Transl, Ger version Bles; 1930

4. Peipert JF: Clinical practice. Genital chlamydial infections. N Engl J Med 2003, 349:2424-2430.

5. Stamm WE: Sexually Transmitted Diseases. 3rd edition. New York: McGraw Hill; 1999:407-422.

6. Mabey D, Peeling RW: Lymphogranuloma venereum. Sex Transm Infect 2002, 78:90-92.

7. Nieuwenhuis RF, Ossewaarde JM, Götz HM, Dees J, Thio HB, Thomeer MGJ, den Hollander JC, Neumann MHA, van der Meijden WI: Resurgence of lymphogranuloma venereum in Western Europe: an outbreak of Chlamydia trachomatis serovar 12 proctitis in The Netherlands among men who have sex with men. Clin Infect Dis 2004, 39:996-1003.

8. Brunelle BW, Nicholson TL, Stephens RS: Microarray-based genomic surveying of gene polymorphisms in Chlamydia trachomatis. Genome Biol 2004, 5:R42

9. Carlson JH, Hughes S, Hogan D, Cieplak G, Sturdevant DE, McClarty G, Caldwell HD, Belland RJ: Polymorphisms in the Chlamydia trachomatis cytotoxin locus associated with ocular and genital isolates. Infect Immun 2004, 72:7063-7072.

10. Read TD, Brunham RC, Shen C, Gill SR, Heidelberg JF, White O, Hickey EK, Peterson J, Utterback T, Berry K, Bass S, Linher K, Weidman J, Khouri H, Craven B, Bowman C, Dodson R, Gwinn M, Nelson W, DeBoy R, Kolonay J, McClarty G, Salzberg SL, Eisen J, Fraser CM: Genome sequences of Chlamydia trachomatis MoPn and Chlamydia pneumoniae AR39. Nucleic Acids Res 2000, 28:1397-1406.

11. Yuan $Y$, Zhang YX, Watkins NG, Caldwell HD: Nucleotide and deduced amino acid sequences for the four variable domains of the major outer membrane proteins of the 15 Chlamydia trachomatis serovars. Infect Immun 1989, 57:1040-1049.

12. Kuo C, Chen WJ: A mouse model of Chlamydia trachomatis pneumonitis. $J$ Infect Dis 1980, 141:198-202.

13. Ito Jl, Lyons JM, Airo-Brown LP: Variation in virulence among oculogenital serovars of Chlamydia trachomatis in experimental genital tract infection. Infect Immun 1990, 58:2021-2023.

14. Carlson JH, Porcella SF, McClarty G, Caldwell HD: Comparative genomic analysis of Chlamydia trachomatis oculotropic and genitotropic strains. Infect Immun 2005, 73:6407-6418.

15. Thomson NR, Holden MTG, Carder C, Lennard N, Lockey SJ, Marsh P, Skipp P, O'Connor CD, Goodhead I, Norbertzcak H, Harris B, Ormond D, Rance R, Quail MA, Parkhill J, Stephens RS, Clarke IN: Chlamydia trachomatis: genome sequence analysis of lymphogranuloma venereum isolates. Genome Res 2008, 18:161-171.

16. Fields KA, Hackstadt T: The chlamydial inclusion: escape from the endocytic pathway. Annu Rev Cell Dev Biol 2002, 18:221-245.

17. Cocchiaro JL, Valdivia RH: New insights into Chlamydia intracellular survival mechanisms. Cell Microbiol 2009, 11:1571-1578.

18. Beagley KW, Huston WM, Hansbro PM, Timms P: Chlamydial infection of immune cells: altered function and implications for disease. Crit Rev Immunol 2009, 29:275-305.

19. Inman RD, Whittum-Hudson JA, Schumacher HR, Hudson AP: Chlamydia and associated arthritis. Curr Opin Rheumatol 2000, 12:254-262.

20. Gérard HC, Krausse-Opatz B, Wang Z, Rudy D, Rao JP, Zeidler H, Schumacher HR, Whittum-Hudson JA, Köhler L, Hudson AP: Expression of Chlamydia trachomatis genes encoding products required for DNA synthesis and cell division during active versus persistent infection. Mol Microbiol 2001, 41:731-741.

21. Patton DL, Kuo CC: Histopathology of Chlamydia trachomatis salpingitis after primary and repeated reinfections in the monkey subcutaneous pocket model. J Reprod Fertil 1989, 85:647-656.

22. Gieffers J, van Zandbergen G, Rupp J, Sayk F, Krüger S, Ehlers S, Solbach W, Maass M: Phagocytes transmit Chlamydia pneumoniae from the lungs to the vasculature. Eur Respir J 2004, 23:506-510. 
23. Koehler L, Nettelnbreker E, Hudson AP, Ott N, Gérard HC, Branigan PJ, Schumacher HR, Drommer W, Zeidler H: Ultrastructural and molecular analyses of the persistence of Chlamydia trachomatis (serovar K) in human monocytes. Microb Pathog 1997, 22:133-142.

24. Schmitz E, Nettelnbreker E, Zeidler H, Hammer M, Manor E, Wollenhaupt J: Intracellular persistence of chlamydial major outer-membrane protein, lipopolysaccharide and ribosomal RNA after non-productive infection of human monocytes with Chlamydia trachomatis serovar K. J Med Microbiol 1993, 38:278-285.

25. Mellman I, Steinman RM: Dendritic cells: specialized and regulated antigen processing machines. Cell 2001, 106:255-258.

26. Pulendran B, Palucka K, Banchereau J: Sensing pathogens and tuning immune responses. Science 2001, 293:253-256.

27. Stagg AJ, Elsley WA, Pickett MA, Ward ME, Knight SC: Primary human T-cell responses to the major outer membrane protein of Chlamydia trachomatis. Immunology 1993, 79:1-9.

28. Lu H, Zhong G: Interleukin-12 production is required for chlamydial antigen-pulsed dendritic cells to induce protection against live Chlamydia trachomatis infection. Infect Immun 1999, 67:1763-1769.

29. Ojcius DM, de Alba Bravo Y, Kanellopoulos JM, Hawkins RA, Kelly KA, Rank RG, Dautry-Varsat A: Internalization of Chlamydia by dendritic cells and stimulation of Chlamydia-specific T cells. J Immunol 1998, 160:1297-1303.

30. Matyszak MK, Young JL, Gaston JS: Uptake and processing of Chlamydia trachomatis by human dendritic cells. Eur J Immunol 2002, 32:742-751.

31. Gervassi A, Alderson MR, Suchland R, Maisonneuve JF, Grabstein KH, Probst P: Differential regulation of inflammatory cytokine secretion by human dendritic cells upon Chlamydia trachomatis infection. Infect Immun 2004, 72:7231-7239.

32. Byrne Gl, Faubion CL: Inhibition of Chlamydia psittaci in oxidatively active thioglycolate-elicited macrophages: distinction between lymphokine-mediated oxygen-dependent and oxygen-independent macrophage activation. Infect Immun 1983, 40:464-471.

33. Shemer $Y$, Sarov I: Inhibition of growth of Chlamydia trachomatis by human gamma interferon. Infect Immun 1985, 48:592-596.

34. Njau F, Wittkop U, Rohde M, Haller H, Klos A, Wagner AD: In vitro neutralization of tumor necrosis factor-alpha during Chlamydia pneumoniae infection impairs dendritic cells maturation/function and increases chlamydial progeny. FEMS Immunol Med Microbiol 2009, 55:215-225.

35. Fehlner-Gardiner C, Roshick C, Carlson JH, Hughes S, Belland RJ, Caldwell HD, McClarty G: Molecular basis defining human Chlamydia trachomatis tissue tropism. A possible role for tryptophan synthase. J Biol Chem 2002, 277:26893-26903.

36. Morrison RP: New insights into a persistent problem - chlamydial infections. J Clin Invest 2003, 111:1647-1649.

37. Caldwell HD, Wood H, Crane D, Bailey R, Jones RB, Mabey D, Maclean I, Mohammed Z, Peeling R, Roshick C, Schachter J, Solomon AW, Stamm WE, Suchland RJ, Taylor L, West SK, Quinn TC, Belland RJ, McClarty G: Polymorphisms in Chlamydia trachomatis tryptophan synthase genes differentiate between genital and ocular isolates. J Clin Invest 2003, 111:1757-1769.

38. Thalmann J, Janik K, May M, Sommer K, Ebeling J, Hofmann F, Genth H, Klos A: Actin re-organization induced by Chlamydia trachomatis serovar D-evidence for a critical role of the effector protein CT166 targeting Rac. PLOS One 2010, 5:e9887.

39. Paul Ehrlich Institute: Notice of Guidelines for Collection of Blood and Blood Components. Volume 62, Volume Volume 62. Bundesministerium der Justiz: Bunndesanzeiger; 2010

40. Wittkop U, Peppmueller M, Njau F, Leibold W, Klos A, Krausse-Opatz B, Hudson AP, Zeidler H, Haller H, Wagner AD: Transmission of Chlamydophila pneumoniae from dendritic cells to macrophages does not require cell-to-cell contact in vitro. J Microbiol Methods 2008, 72:288-295.

41. Livak KJ, Schmittgen TD: Analysis of relative gene expression data using real-time quantitative PCR and the 2(-Delta Delta $C(T)$ ) Method. Methods 2001, 25:402-408

42. Schnitger K, Njau F, Wittkop U, Liese A, Kuipers JG, Thiel A, Morgan MA, Zeidler H, Wagner AD: Staining of Chlamydia trachomatis elementary bodies: a suitable method for identifying infected human monocytes by flow cytometry. J Microbiol Methods 2007, 69:116-121.

43. Beatty WL, Belanger TA, Desai AA, Morrison RP, Byrne Gl: Tryptophan depletion as a mechanism of gamma interferon-mediated chlamydial persistence. Infect Immun 1994, 62:3705-3711.
44. Njau F, Geffers R, Thalmann J, Haller H, Wagner AD: Restriction of Chlamydia pneumoniae replication in human dendritic cell by activation of indoleamine 2,3-dioxygenase. Microbes Infect 2009, 11:1002-1010.

45. Dessus-babus S, Darville TL, Cuozzo FP, Ferguson K, Wyrick PB: Differences in innate immune responses ( in vitro ) to HeLa cells infected with nondisseminating serovar $\mathrm{E}$ and disseminating serovar L2 of Chlamydia trachomatis. Infect Immun 2002, 70:3234-3248.

46. Grohmann U, Fallarino F, Puccetti P: Tolerance, DCs and tryptophan: much ado about IDO. Trends Immunol 2003, 24:242-248.

47. Akira S, Takeda K: Toll-like receptor signalling. Nat Rev Immunol 2004, 4:499-511.

48. Manor E, Sarov l: Fate of Chlamydia trachomatis in human monocytes and monocyte-derived macrophages. Infect Immun 1986, 54:90-95.

49. Beatty WL, Morrison RP, Byrne Gl: Persistent chlamydiae: from cell culture to a paradigm for chlamydial pathogenesis. Microbiol Rev 1994, 58:686-699.

50. Wolf $\mathrm{K}$, Fischer $\mathrm{E}$, Hackstadt T: Degradation of Chlamydia pneumoniae by peripheral blood monocytic cells. Infect Immun 2005, 73:4560-4570.

51. Sommer K, Njau F, Wittkop U, Thalmann J, Bartling G, Wagner A, Klos A: Identification of high- and low-virulent strains of Chlamydia pneumoniae by their characterization in a mouse pneumonia model. FEMS Immunol Med Microbiol 2009, 55:206-214.

52. Medzhitov $R$, Janeway C: Innate immune recognition: mechanisms and pathways. Immunol Rev 2000, 173:89-97.

53. Hemmi H, Takeuchi O, Kawai T, Kaisho T, Sato S, Sanjo H, Matsumoto M, Hoshino K, Wagner H, Takeda K, Akira S: A Toll-like receptor recognizes bacterial DNA. Nature 2000, 408:740-745.

54. Ozinsky A, Underhill DM, Fontenot JD, Hajjar AM, Smith KD, Wilson CB, Schroeder $L$, Aderem $A$ : The repertoire for pattern recognition of pathogens by the innate immune system is defined by cooperation between toll-like receptors. Proc Natl Acad Sci USA 2000, 97:13766-13771.

55. Muzio M, Ni J, Feng P, Dixit VM: IRAK (Pelle) family member IRAK-2 and MyD88 as proximal mediators of IL-1 signaling. Science 1997, 278:1612-1615.

56. Kawai T, Adachi O, Ogawa T, Takeda K, Akira S: Unresponsiveness of MyD88-deficient mice to endotoxin. Immunity 1999, 11:115-122.

57. Hoebe K, Du X, Georgel P, Janssen E, Tabeta K, Kim SO, Goode J, Lin P, Mann N, Mudd S, Crozat K, Sovath S, Han J, Beutler B: Identification of Lps2 as a key transducer of MyD88-independent TIR signalling. Nature 2003, 424:743-748.

58. Agrawal T, Bhengraj AR, Vats V, Salhan S, Mittal A: Expression of TLR 2, TLR 4 and iNOS in cervical monocytes of Chlamydia trachomatis-infected women and their role in host immune response. Am J Reprod Immunol 2011, 66:534-543.

59. Darville T, Hiltke TJ: Pathogenesis of genital tract disease due to Chlamydia trachomatis. J Infect Dis 2010, 201(2):S114-S125.

doi:10.1186/s12866-014-0209-3

Cite this article as: Datta et al:: Differential infection outcome of Chlamydia trachomatis in human blood monocytes and monocyte-derived dendritic cells. BMC Microbiology 2014 14:209.

\section{Submit your next manuscript to BioMed Central and take full advantage of:}

- Convenient online submission

- Thorough peer review

- No space constraints or color figure charges

- Immediate publication on acceptance

- Inclusion in PubMed, CAS, Scopus and Google Scholar

- Research which is freely available for redistribution 